\title{
Effects of the microbiome manipulation on survival and GI tract development of larval zebrafish (Danio rerio)
}

\author{
Uthpala Padeniya ${ }^{1}$, Shafira Septriani ${ }^{1}$, Arjay Pataueg ${ }^{1}$, Christopher L. Brown ${ }^{1 *}$ \\ ${ }^{1}$ World Fisheries University Pilot Programme, Pukyong National University (PKNU), Busan, South \\ Korea
}

\begin{abstract}
Microbial diversity within an aquatic community can be used to increase the growth and development of organisms. In this study, Zebrafish larvae were reared in three treatments 1) a probiotic containing 17 strains of Lactobacillus spp., 2) an amoxicillin solution, 3) water from the broodstock culture tank as the control. Survival of the larvae throughout $10 \mathrm{dpf}$ was recorded. To determine the development of the gastrointestinal tract DASPEI stain was used for larvae at the age of $3 \mathrm{dpf}$ to $6 \mathrm{dpf}$. The intensity of the fluorescence in each larva was observed through the automated digital microscope. According to the experiment results, a significant difference $(\mathrm{P}<0.0001)$ in the survival rates among all treatments was recorded. The probiotic-treated larvae (PTL) had a higher survival rate. This could be due to the presence of lactic acid bacteria in the probiotic treatment, which helps in enhancing immunity. In DASPEI staining, also PTL exhibits more fluorescence in the GI tract at 24 hours to 48 hours post-hatch than the other two treatments. The main possible reason behind this could also be the presence of Lactobacillus spp. Which directly influences the higher activity of the digestive system.
\end{abstract}

\section{Introduction}

Aquaculture being the fastest growing food-producing industry globally, it is now developing and expanding worldwide. The popularity and demand for aquatic food among people are to increase. Because capture fisheries have leveled off, aquaculture has been considered the only means to satisfy the world's growing demands. Growth of this industry influence so many other factors like trade and consumption preferences [1]. For the growth of this sector, the fish should be in good health with better feed utilization and a high survival rate. This directly influences the cost of production, both lowering it and producing the best marketable size and quality of fish [2]. Manipulation of fish microbiota to improve the quality and growth of aquatic organisms can be of great advantage. The digestive tract of fish is host to several beneficial microbes from surrounding water and food, benefiting the growth, disease resistance, and survival of fish. Correlations between the microbial community and its effects

\footnotetext{
*Corresponding author: brownchristopher38@gmail.com
} 
on fish development have been proposed and scholarly research interest in this subdiscipline has grown [3].

\subsection{Microbiome}

The collection of all different microorganisms in a specific area with their genomes together and interactions with one another is typically known as a microbiome, according to Nobel laureate Joshua Lederberg and others [4]. These include not only bacteria but also fungi, algae, protozoans, and viruses. Microbiomes influence health and physiology profoundly, ranging from the most advanced species into the least advanced species, including plants. They can alter the host physiology, enabling disease resistance and defense, and contribute to nutrient processing and absorption, resulting in improved growth and development [5]. The microbiome can consist of both pathogenic and advantageous species. In a healthy environment, these pathogenic and benign microbes are living symbiotically. They are wellbalanced, but in response to various environmental factors, this balance can be disrupted, causing diseases and various harmful effects to the host organism carrying these microbiomes $[6]$.

\subsubsection{Aquaculture and microbiome}

This sort of manipulation of the microbiome is a relatively new and untested concept. Correct microbial management can prevent the spread of pathogens and maintain good water quality in aquacultural practices. It can also be manipulated, so that antibiotic application is reduced. To assess the status of the microbiome, proper identification of healthy and pathogenic communities should be made [7]. Microbial intervention is a new approach to sustainable fish production and controlling the spread of disease environmentally friendly. The primary method of microbial manipulation is done by augmentation or introduction of beneficial microbes, known as probiotics. Probiotics are delivered into the body as a form of diet or by immersion. This is done to improve their welfare and nutrition. One of the most commonly used probiotics is the lactic acid class of bacteria, or LA bacteria, in larviculture [2]. These bacteria colonize in the intestines and can assist in resisting low $\mathrm{pH}$ and bile salts. The application method of probiotics came because LA bacteria are not typically present in sufficient amounts [8].

\subsection{Zebrafish and it's microbiota}

Danio rerio belongs to the family Cyprinidae and is a freshwater fish native to south and Southeast Asia. They are small fish with longitudinal stripes. An adult fish may weigh about 0.5 to $0.9 \mathrm{~g}$ with a total length of about 22 to $38 \mathrm{~mm}$ [9]. Zebrafish as model organisms have mainly influenced the research areas in aquaculture, and the field has contributed to the industry's development [10].

Studies on the larval intestine of D. rerio have found that the most common genera are Aeromonas, Pseudomonas and Vibrio [11]. At the beginning of the lifecycle, embryos within eggs develop in a sterile condition, but after hatching, they encounter microbes in the environment. Then the development starts by maintaining a close relationship with the microorganisms. The diversity of microbiota changes and becomes more complex with growth and development [12]. The zebrafish gut microbiome differs slightly between wild and laboratory-reared fish. According to Roselers et al., [13] 21 Operational Taxonomical Units (OTUs) were similar to wild and facility reared fish. This evidence strongly suggests that in zebrafish, a core microbiota is present. The bacterial phylum Proteobacteria is the most common throughout each stage of the lifecycle. Many external factors can influence the 
composition of the microbiome. They can be either increase the diversity like probiotics or disrupt the composition. Several chemicals containing nanoparticles, Bisphenol A (BPA), and antibiotics have demonstrated antimicrobial effects that disturb the microbiome's diversity in zebrafish [11].

\section{Methodology}

\subsection{Experimental design}

Adult wild-type zebrafish needed for the experiment were obtained from Jaloo commercial ornamental fish farm (Jwabu-dong, Chungnam, South Korea) and held in an indoor recirculating system at Pukyong National University (PKNU). Animal housing, care, and experimental procedures followed the Institutional Animal Care and Use Committee of PKNU. TetraMin ${ }^{\circledR}$ Tropical Flakes (Spectrum Brands Pet, Blacksburg, USA) and newly hatched Artemia salina nauplii (INVE Aquaculture cysts, Nonthaburi, Thailand) were given twice a day as feed. The light: dark cycle was held at 14L: 10D. The temperature was maintained at $24^{\circ} \mathrm{C} \pm 1^{\circ}$. Female and male adult fish were held separately in two tanks. Spawning was done in groups of six to obtain off springs naturally, where the female to male ratio was $4: 2$, respectively. Fertilized eggs were collected the next day before noon $[9,14$, $15]$.

Three treatments were prepared with water from the broodstock tank for experimental treatment of embryos collected from the hatchery. Each treatment had six replicates with ten eggs per each. Fertilized eggs were immersed in solutions as described below.

1. Control - A culture medium consisting of untreated water drawn from the broodstock tank served as a control.

2. Probiotic - Hurum daily live probiotics 104 cells $/ \mathrm{ml}$, containing Lactobacillus acidophilus, Enterococcus faecium, 17 Multi-Strain Lactobacillus. Hurum is a commercially available probiotic product for human consumption, containing various live bacterial species commonly used in aquaculture. Most of these are classified as lactic acid bacteria (LAB) - Gram-positive bacteria that produce lactic acid as a metabolic product of fermentation. Some of these species occur naturally in the freshwater fish intestine, and some are used as probiotics for beneficial health effects.

3. Antibiotic $-0.01 \mathrm{mg} / \mathrm{ml}$ Amoxycillin dissolved in culture water. Amoxycillin is a semisynthetic derivative of penicillin with a broad spectrum of bactericidal actions. The dose selected for this study follows the recommended concentration of live bacteria for probiotic actions on zebrafish larvae [16].

Each day the groups were carefully observed and monitored. Any embryos or larvae subject to mortality were removed. $50 \%$ of the culture media was replaced daily. From day eight, they were fed with water containing an infusoria culture consisting largely of rotifers and Paramecium sp. [17]. Some monocellular algae were also seen in our infusoria cultures.

\subsection{Survival rates}

Rates of survival were quantified for ten days post fertilization (dpf). The heartbeat of both the embryos and larvae were observed in the LEICA DMI6000 automated digital microscope to ensure they were alive or dead. 


\subsection{DASPEI staining}

For the examination of morphological development of larvae especially the gastrointestinal track development DASPEI (4-(Dimethylamino)styryl)-N-ethylpyridiniumiodide) stain was used. Three larvae of $3 \mathrm{dpf}$ ( 1 days post-hatch) to $6 \mathrm{dpf}$ (4 days post-hatch) from each treatment were immersed for three hours in $6 \mathrm{ppm}$ of the stain and were examined under fluorescence at 12 hours each from the automated digital microscope (LEICA DMI6000) [18].

\subsection{Statistical analysis}

The morphometric parameters were analyzed using a two-way analysis of variance (SPSS), with treatment and age being the factors. Normality and homogeneity of the sample values were conducted by using SPSS. When normality failed, data were transformed normalized. Differences in the parameters among the treatments were considered. The significance was taken when $\mathrm{P}<0.05$ [19]. Using the GraphPad Prism 9 software, the graphs were drawn.

\section{Results}

\subsection{Rates of survival}

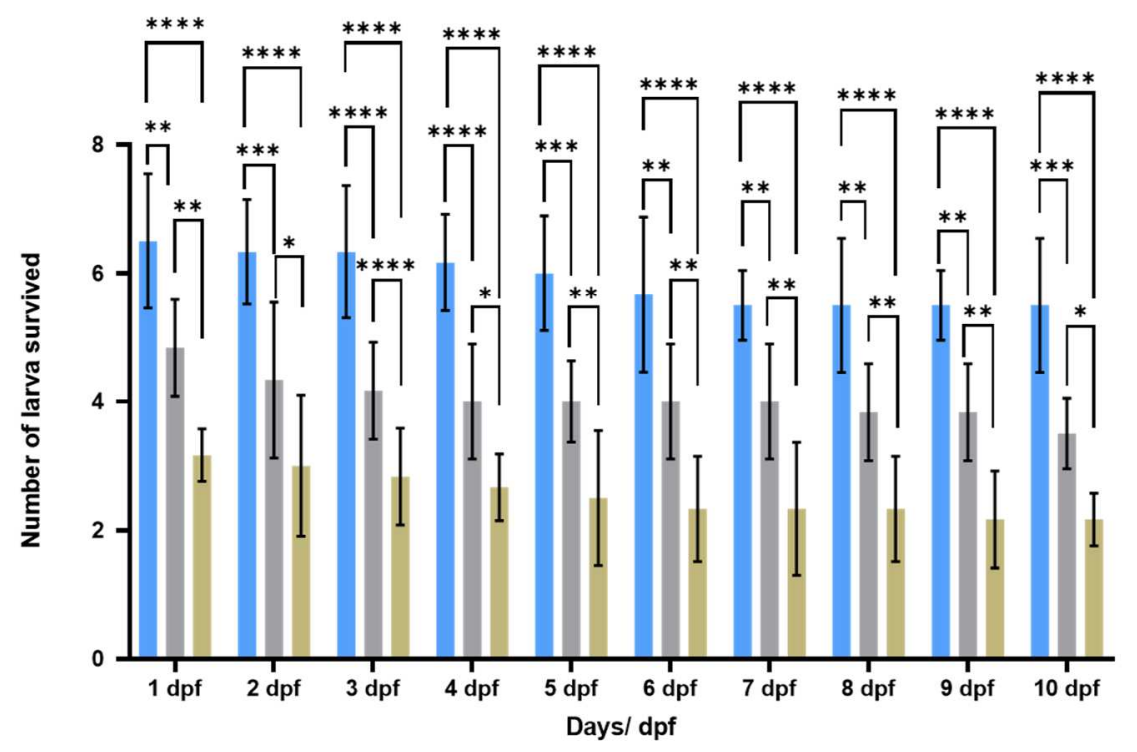

Fig. 1. Survival rate of larvae after 10 days post fertilization.

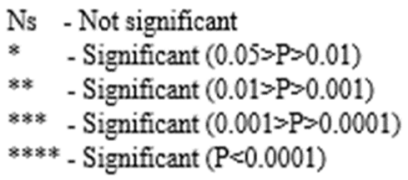


The mean survival rates of larvae were $59 \%, 40 \%$, and $25 \%$ in probiotic control and antibiotic-treated larvae, respectively. A higher significance $(\mathrm{P}<0.0001)$ was recorded throughout the probiotic and antibiotic treatments throughout the period. However, the significance between the probiotic and the control was lower than this. The figures show that the survival rates are higher in probiotic-treated larvae even before the first feeding.

\subsection{DASPEI staining}

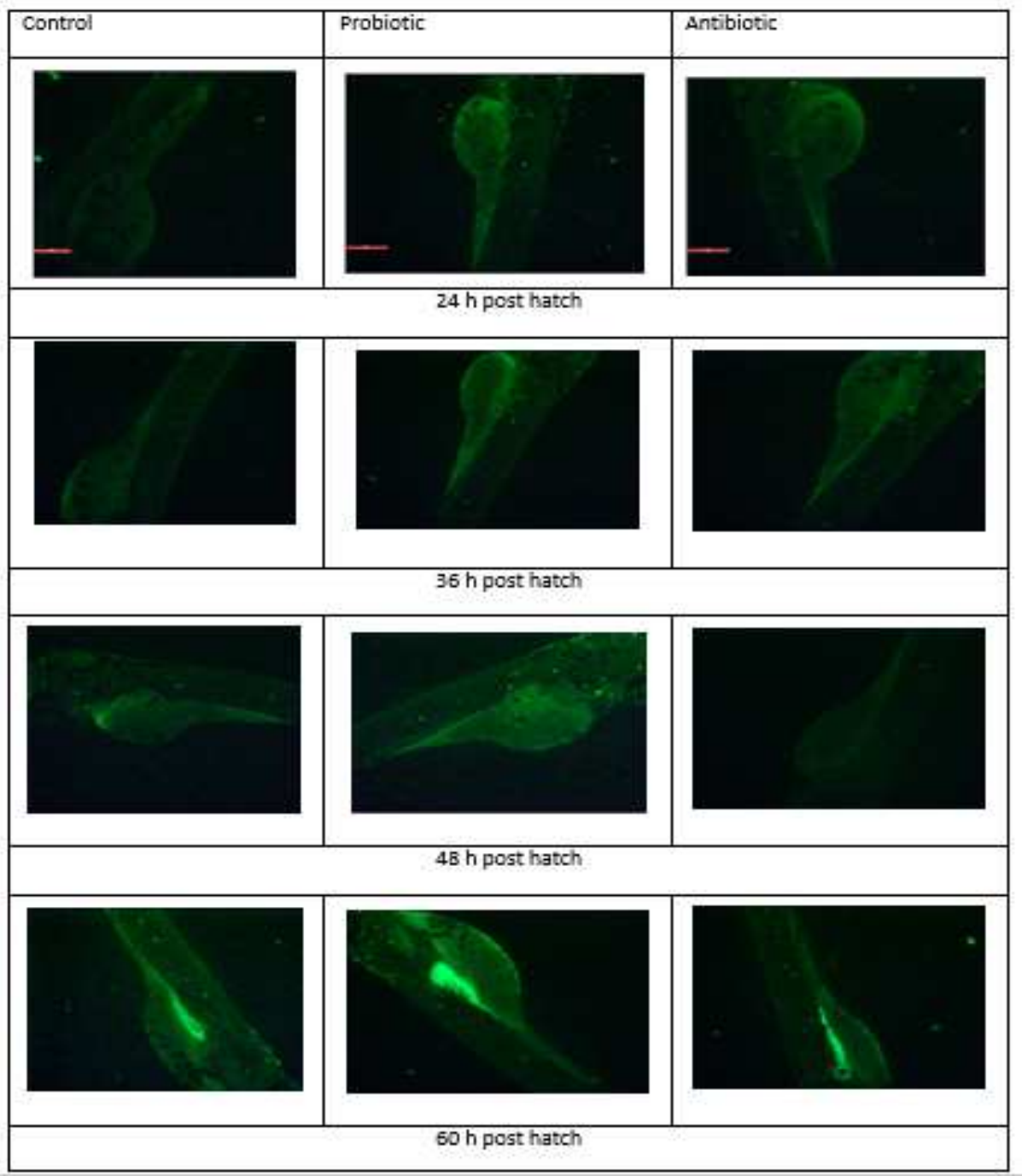

Fig. 2. Florescent microscopical images of the gastrointestinal tract of zebrafish larvae in different treatments after staining with DASPEI.

Through a thorough examination of these images, some clear patterns are evident. In all treatments, the fluorescence of the GI tract increases gradually with the increase of the period.

In the probiotic-treated larvae, the GI tract in 24 hours post-hatch exhibit fluorescence more than antibiotic and control. In $36 \mathrm{~h}$ and $48 \mathrm{~h}$ post-hatch larvae, this condition is more 
visible, that it shows more fluorescence in the probiotic treated larvae. After $60 \mathrm{~h}$ post-hatch larvae in all three treatments, the GI tract is fully developed. However, the track is undergoing much fluorescence in probiotic-treated larvae than the other two treatments.

\section{Discussion}

This study was conducted to understand how microbiota in fish embryos and larvae influence its survival and GI tract development. For the identification of larval fish microbiota on growth, zebrafish was used as the model organism. Zebrafish are aquatic organisms with many relationships and associations with microorganisms from their ambient water [20].

The high survival rates of probiotic-treated larvae (PTL) may probably reflect the improved health and immunity with microbiota manipulation. The positive alterations done to the microbiota, like the addition of probiotics, positively influences their survival rates than the negative alterations done, such as the addition of antibiotics. The larval fish does not have a proper sophisticated immune system. Hence, the commensal microbiota in surrounding water and on the body surface acts as the first line of defense, which is the innate immune system [21]. These environmental bacteria produce immune activities and also maintain mucosal tolerance. This is why the PTL and control-treated larvae (CTL) showed better survival rates [22]. As the balance of the harmful and beneficial bacteria is disrupted, the mortalities increased in antibiotic-treated larvae (ATL). Also, as there was no bacterial colonization inside the larvae, mortalities throughout the period could be seen.

The most important conclusion is that the presence of lactic acid bacteria in probiotic treatment was probably the cause for high survival rates. These LAB produce several extracellular products such as lactic acid, hydrogen peroxide, carbon dioxide, antibiotic peptides, etc., against pathogenic bacteria and enhance immunity. Also, it is believed that probiotics and the bacteria they contain may interact with gut-associated lymphoid tissue (GALT) and induce their effects on immunity. In fish, these GALTs are very low, but other defenses like macrophages, granulocytes IgM are found in the intestines of freshwater fish [23].

The development of the gut happens progressively with the organization and polarization of the columnar epithelium. The rostral gut develops first and then the hindgut followed by the midgut, similar to that of mammals. The opening of the zebrafish mouth first occurs after 72 hours post-fertilization. By this time, the digestive tract appears as a continuous tube. Though the embryos develop in a microbial-free environment, the larval intestine becomes colonized by bacteria after hatching [24].

From the results of the DASPEI staining, it was observed that in PTL, there was an accelerated onset of gut fluorescence from the rostral end followed by hind and midgut than the other two larvae types. This fluorescence due to DASPEI suggests functional mitochondria in the gut, and the intestine is active indigestion. However, by the end of 60 hours post-hatch, the intestinal tract of all the three larval types was fully developed. The main possible reason for the PTL to show more fluorescence is the presence of Lactobacillus spp. and the high abundance of other bacteria in the intestinal tract. Many studies have suggested that microbiota influence the cell fate decisions in the gut epithelium [25]. Also, the microbiota can influence the AP activity (which is a classic marker of enterocyte maturation) in the intestines of zebrafish [26]. The probiotic mixture, which contains Lactobacillus rhamnosus modulate the gut microbiome of zebrafish to a great extent by reducing the abundance of actinobacteria. Furthermore, they will increase the microvilli content and the lengths of enterocytes which will raise the activity and functions of the intestine [27]. Manipulation of the microbiome by the introduction of Lactobacillus spp. Directly influences the higher activity of the digestive system, thereby contributing to an increase in the growth of fish. 


\section{Conclusion}

This study shows that the correct manipulation of microbiota can lead to various benefits for the growth and survival of the early stages of fish's life. They have a close association with the surrounding environment and its microbial community. The microbes living in water, on the surface, and inside the fish larvae provide protection, increase development, and provide nutrition [28-29]. Additionally, increased functionality of the intestines was statistically significantly enhanced, and relative to the other treatments were also observed. Correct manipulation of the microbiota will help reduce antibiotics in aquacultural practices and can be used to promote the health of fish. However, the larvae in the control treatment too had considerable survival rates. All these positive changes were mainly seen before the first feeding due to probiotics containing Lactobacillus spp. Into the surrounding environment. Poor growth and development were seen in antibiotic-treated larvae with high mortalities, serving as a clear indication that the ambient microbiota and those in colonies inside the larvae are crucial for their development.

\section{References}

1. R. Subasinghe, D. Soto, J. Jia, Rev. Aquac., 1, 1 (2009)

2. G. Burr, D. Gatlin III, S. Ricke, J. World Aquacult. Soc., 36, 4 (2005)

3. M. Ghanbari, W. Kneifel, K. J Domig, Aquac. Res. 448 (2015)

4. S. L. Prescott, J. Hum. Microbiome, 4, 24-25 (2017)

5. E. Ringø, H.V. Doan, S.H. Lee, M. Soltani, S.H. Hoseinifar, R. Harikrishnan, S.K. Song, J. Appl. Microbiol., 129, 116-136 (2020)

6. D. Rybakova, R. Mancinelli, M. Wikström, A.S. Birch-Jensen, J. Postma, R.U. Ehlers, G. Berg, Microbiome, 5, 1 (2017)

7. K.K. Dittmann, B.B. Rasmussen, M. Castex, L. Gram, M. Bentzon-Tilia, Microb. Biotechnol., 10, 6 (2017).

8. L. Gram, E. Ringø, Elsevier Science Publisher, 2(2005)

9. C. Lawrence, T. Mason, ILAR J., 53, 2 (2012)

10. L. Ribas, F. Piferrer, Rev. Aquac., 6, 4 (2014)

11. K. Stagaman, T. J. Sharpton, K. Guillemin, Lab. Anim., 49, 7 (2020)

12. W. Z. Stephens, A. R. Burns, K. Stagaman, S. Wong, J.F. Rawls, K. Guillemin, B.J Bohannan, ISME J., 10, 3 (2016)

13. E. K. Roeselers, W. Z. Mittge, D.M. Stephens, C.M. Parichy, K. Cavanaugh, K. Guillemin, J.F. Rawls, ISME J., 5, 10 (2011)

14. Z.M. Varga, Meth. Cell Biol., 104, 453-478 (2011)

15. M.H.R. Molla, M.T. Hasan, W. J. Jang, C.D.S. Diaz, P. Appenteng, H. Marufchoni, B. Jahan, and C.L. Brown, Aquac. Res., 50, 11 (2019)

16. S. Falcinelli, A. Rodiles, S. Unniappan, S. Picchietti, G. Gioacchini, D.L. Merrifield, O. Carnevali, Sci. Rep., 6, 18061 (2016).

17. P. Siriyappagouder, J. Galindo-Villegas, J. Lokesh, V. Mulero, J.M. Fernandes, V. Kiron, Front. Microbiol., 9, 1868 (2018)

18. C.L Brown, B.G. Kim, Aquac. Res., 135, 1-3 (1995)

19. P.R. Biga, F.W. Goetz, Am. J. Physiol. Regul. Integr. Comp. Physiol., 291, 5 (2006)

20. A.R. Burns, K. Guillemin, Curr. Opin. Microbiol., 38 (2017)

21. J.A. Olafsen, Aquac. Res, 200 (2001)

22. I. de Bruijn, Y. Liu, G.F. Wiegertjes, J.M. Raaijmakers, FEMS Microbiol. Ecol., 94, 1 (2018)

23. A. Dimitroglou, D.L. Merrifield, O. Carnevali, S. Picchietti, M. Avella, C. Daniels, S.J. Davies, Fish Shellfish Immunol., 30, 1 (2011) 
24. V.J. McCracken, R.G. Lorenz, Microrev. Cell. Microbiol., 3, 1 (2001)

25. A. Uribe, M. Alam, O. Johansson, T. Midtvedt, E. Theodorsson, Gastroenterology, 107, $5(1994)$

26. J.M. Bates, E. Mittge, J. Kuhlman, K.N. Baden, S.E. Cheesman, K. Guillemin, Dev. Biol., 297, 2 (2006)

27. S. Falcinelli, A. Rodiles, S. Unniappan, S. Picchietti, G. Gioacchini, D.L. Merrifield, O. Carnevali, Sci. Rep., 6, 18061 (2016).

28. A. Gildberg, H. Mikkelsen, E. Sandaker, E. Ringø, Hydrobiologia, 352 (1997)

29. E. Ringø, L. Løvmo, M. Kristiansen, Y. Bakken, I. Salinas, R. Myklebust, T.M. Mayhew, Aquac. Res., 41, 4 (2010) 